\title{
Analysis of the Influence of the Disturbance on Bacterial Colony by Stochastic Reaction Diffusion Equations
}

\author{
Masaaki ISHIKAWA ${ }^{\dagger}$, Keiichi MIYAJIMA ${ }^{\dagger}$ and Takayuki TANABE ${ }^{\ddagger}$ \\ ${ }^{\dagger}$ Department of Computer Science and Systems Engineering \\ Faculty of Engineering, Yamaguchi University

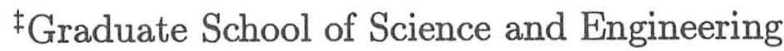 \\ Yamaguchi University \\ †, $¥$ 2-16-1 Tokiwadai, Ube, Yamaguchi, 755-8611 Japan \\ e-mail: ishikawa@csse.yamaguchi-u.ac.jp \\ Tel \& Fax: +81 836859524
}

\begin{abstract}
This paper is concerned with a mathematical modeling of the bacterial colony formations by stochastic reaction diffusion equations. It is well known that the bacteria called Bacillus subtilis create several kinds of colony pattern depending on environmental situations. We often observe many patterns besides bacterial colony patterns: spatiotemporal patterns such as target and spiral patterns in the BZ reaction are observed in the various fields of engineering, including fluid and chemical engineering. The analysis of such patterns is one of important problems of engineering. In this paper, focusing our attention on the bacterial colony patterns, the stochastic modeling of behaviors of the bacteria called Bacillus subtilis, analysis of the created patterns and influence of disturbance on the colony patterns are studied by numerical simulations.
\end{abstract}

Keywords: Bacterial colony, pattern formations, reaction-diffusion systems, stochastic systems, numerical simulations

\section{Introduction}

It is well known that Bacillus subtilis [1]-[3] creates five kinds of patterns depending on both the concentrations of nutrient and substrate(agar), $C_{n}$ and $C_{a}$ as shown in Fig.1 (quoted from [1]). The vertical and horizontal axes in Fig. 1 denote $C_{n}$ and $1 / C_{a}$ respectively. Depending on values of $C_{n}$ and $C_{a}$, the bacterial colony pattern becomes a dendritic one as diffusion limited aggregation(DLA) in the region
$A$ (low $C_{n}$ and high $C_{a}$ ), roughly round shape with rough envelope(Eden-like pattern [1]) in the region $B$ (high $C_{n}$ and high $C_{a}$ ), concentric-ring(CR) in the region $C$, homogeneous spreading $\operatorname{disk}(\mathrm{HSD})$ in the region $D$ and dense branching morphology (DBM) in the region $\mathrm{E}$.

In this paper, taking the random fluctuation of population variation of bacteria into consideration, we propose the stochastic reaction diffusion system as the model which can create various bacterial colony 
patterns as shown in Fig.1. And the effectiveness of the proposed model is studied by simulations.

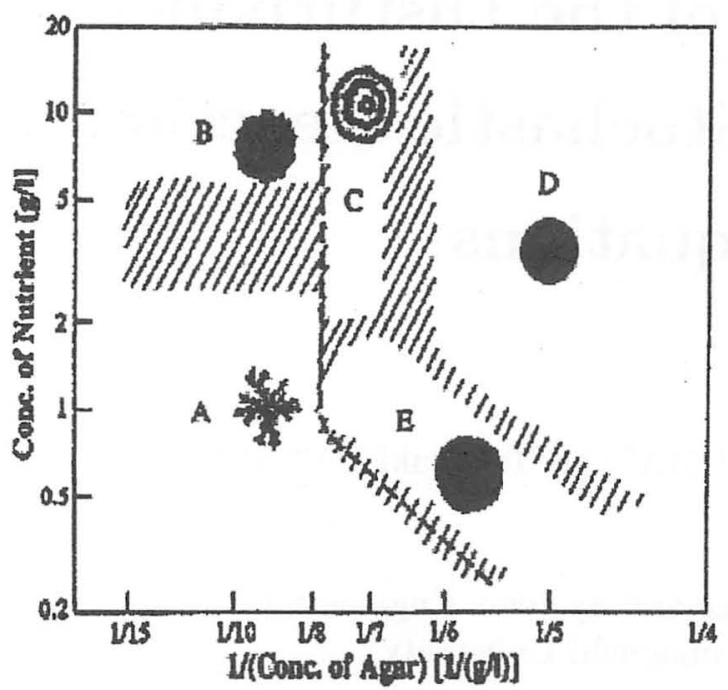

Figure 1: Morphological phase diagram of bacterial colony(quoted from [1])

\section{Stochastic Modeling of Bac- terial Colony Formations}

\subsection{Stochastic Model I and Simula- tions}

It is considered from experimental observations that there exist two types of bacteria, one is the active bacteria, which move and grow, the other is the inactive bacteria, which do not move and grow. Now let $u(t, x), w(t, x)$ and $v(t, x)$ be the population densities of active and inactive bacteria and the concentration of nutrient at time $t$ and position $x$ respectively. Taking fluctuations in the population density of the active bacteria into consideration, we propose the stochastic reaction diffusion model:

$$
\begin{gathered}
\frac{\partial u(t, x)}{\partial t}=d_{u} \Delta u(t, x)+\alpha u(t, x) v(t, x) \\
-q(u, v) u(t, x)+c(x) u(t, x) \frac{\partial z(t, x)}{\partial t} \\
(t, x) \in \Theta \times G \\
\frac{\partial v(t, x)}{\partial t}=d_{v} \Delta v(t, x)-\alpha u(t, x) v(t, x) \\
(t, x) \in \Theta \times G \\
\frac{\partial w(t, x)}{\partial t}=q(u, v) u(t, x) \quad(t, x) \in \Theta \times G
\end{gathered}
$$

where $\Theta=(0, T), G \subset R^{2}, \Delta(\cdot)$ is a Laplacian, $z(t, x)$ is a Wiener process [4] with respect to time with an incremental covariance operator $Q, \alpha$ is a positive constant and the function $q(u, v)$ denotes the conversion rate from the active bacteria to the inactive one.

From experimental observations [2], it is confirmed that the conversion rate $q$ from the active bacteria to inactive one is monotonically decreasing with respect to the population density $u$ of active bacteria and the concentration $v$ of nutrient. So, according to [2], we set $q(u, v)$ as

$$
q(u, v)=\frac{1}{\left(1+\frac{u}{a_{1}}\right)\left(1+\frac{v}{a_{2}}\right)}
$$

where $a_{1}$ and $a_{2}$ are positive constants.

It should be noted that the inactive bacteria that convert from active ones never become active ones again as long as the food is not given from the outside. And the inactive bacteria do not move and grow, so the diffusion term does not appear in (3). The initial and boundary conditions are given by

$$
\begin{aligned}
u(0, x) & =u_{0}(x)>0, \quad x \in G \\
v(0, x) & =v_{0}(x)>0, \quad x \in G \\
w(0, x) & =0, \quad x \in G \\
\frac{\partial u(t, x)}{\partial \nu} & =\frac{\partial v(t, x)}{\partial \nu}=0, \quad(t, x) \in \Theta \times \Gamma
\end{aligned}
$$

where $\Gamma$ denotes the boundary of $G$ and $\partial(\cdot) / \partial \nu$ is an exterior normal derivative on $\Gamma$.

Now, by simulations, consider whether the proposed model (1) to (8) can create the bacterial colony patterns as shown in Fig.1.

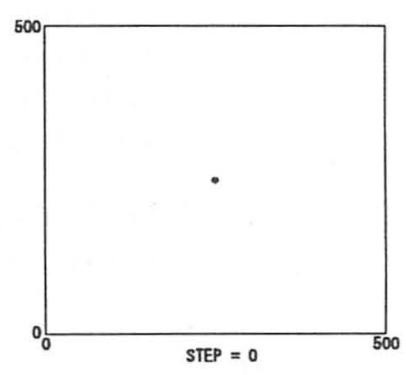

Figure 2: Initial value

Setting as $G=(0,1) \times(0,1)$ and $d_{v}=1.0, a_{1}=$ $1 / 2400, a_{2}=1 / 120, u_{0}(x, y)=1(x=0.5, y=$ $0.5), 0(x \neq 0.5, y \neq 0.5)$ (see Fig. 2), $\alpha=1, c=$ 0.03 and changing values of $d_{u}$ and $v_{0}$ as below, simulations are performed.

$\left(\right.$ Case-1) $d_{u}=0.2, v_{0}=0.2$ 
(Case-2) $d_{u}=0.05, v_{0}=0.087$

(Case-3) $d_{u}=0.12, v_{0}=0.071$

$\left(\right.$ Case-4) $d_{u}=0.05, \quad v_{0}=0.1$

It should be noted that Cases 1 to 4 are corresponding to the regions $D, A, E$ and $C$ in Fig. 1 respectively.

In each figure of simulation results, scales of horizontal and vertical axes show the segmentation of the region $(0,1)$ into 500 intervals. The bacterial colony patterns $u+w$ under noise in Cases 1 to 4 are shown in Figs. 3 to 6 . From results of simulations, we can say that patterns of bacterial colony change by the environmental conditions, i.e., nutrient concentration and the hardness of the agar and all created patterns are similar to experimental results in Fig.1. On the other hand, the deterministic model(i.e., $c=0$ in (1)) only generates the artifial pattern, for example, such as Fig.7 which corresponds to Case-4(Fig.6) under no noise. The envelope of concentric circle is very smooth, which does not seem the observed CR pattern. Therefore, the proposed stochastic model seems to be appropriate as the model for the generation of the bacterial colony.

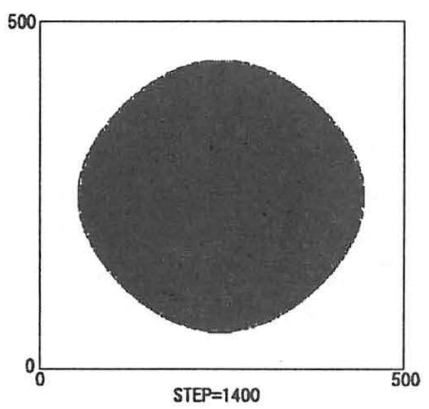

Figure 3: Disk pattern under noise (Case-1)

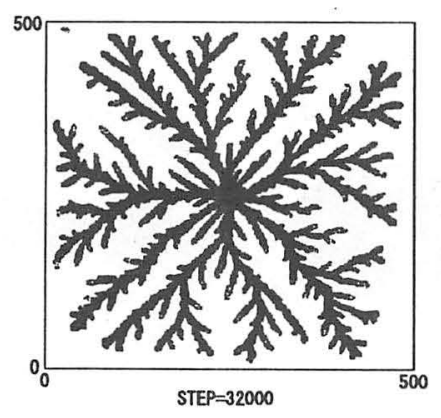

Figure 4: DLA pattern under noise (Case-2)

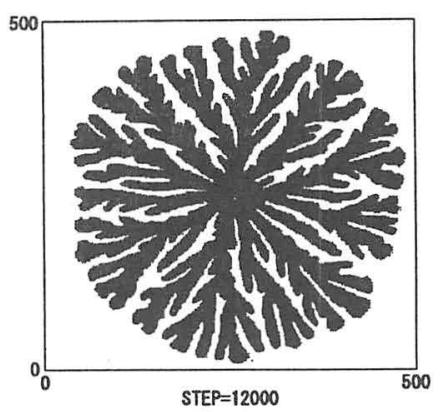

Figure 5: DBM pattern under noise (Case-3)

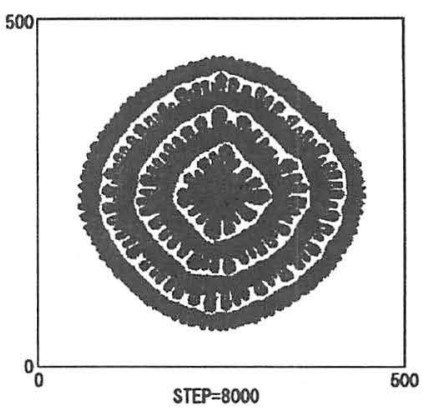

Figure 6: CR pattern under noise (Case-4)

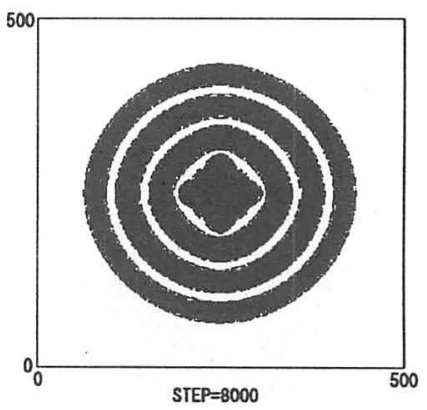

Figure 7: CR pattern under no noise (Case-4)

Since the proposed model (1) to (8) can create the colony patterns $\mathrm{A}, \mathrm{C}, \mathrm{D}$ and $\mathrm{E}$ as shown in Fig.1, however, the model I cannot generate the Eden-like pattern B in Fig.1, in the sequel, consider the model which can genarate the Eden-like pattern.

\subsection{Stochastic Model II and Simula- tions}

In (1), revising the diffusion term in such a way that $d_{u} \Delta u \rightarrow d_{u} \nabla(u \nabla u)$, i.e., assuming the diffusion coefficient depends on the density of the active bacteria, we propose a new model:

$$
\frac{\partial u(t, x)}{\partial t}=d_{u} \nabla(u(t, x) \nabla u(t, x))+\alpha u(t, x) v(t, x)
$$




$$
\begin{gathered}
-q(u, v) u(t, x)+c(x) u(t, x) \frac{\partial z(t, x)}{\partial t} \\
(t, x) \in \Theta \times G \\
\frac{\partial v(t, x)}{\partial t}=d_{v} \Delta v(t, x)-\alpha u(t, x) v(t, x) \\
(t, x) \in \Theta \times G \\
\frac{\partial w(t, x)}{\partial t}=q(u, v) u(t, x) \quad(t, x) \in \Theta \times G
\end{gathered}
$$

with the initial and boundary conditions (5) to (8). Next, consider simulation experiments by the model (9) to (11).

Setting as $G=(0,1) \times(0,1), d_{u}$ and $v_{0}$ as below and letting other parameters be same as Cases-1-to 4 , simulations are performed.

(Case-5) $d_{u}=0.2, v_{0}=0.4$

The created pattern is shown in Fig.8. Figures 8(a) and $8(\mathrm{~b})$ correspond to cases under noise and no noise respectively. In the case of no noise, the disk like pattern is created. On the other hand, under noise, Eden-like pattern is generated as shown in Fig.8(a). This result says that the proposed stochastic model is very useful for generation of the bacterial colony pattern B in Fig.1, which is not generated by the Model I(Eq. (1) to (3)).

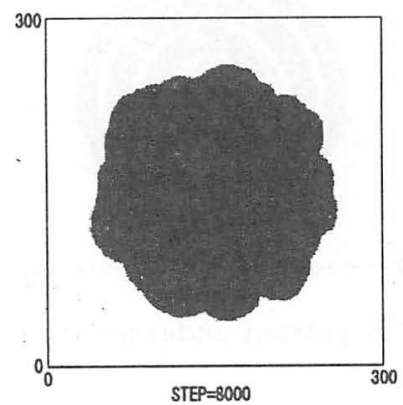

(a) With noise

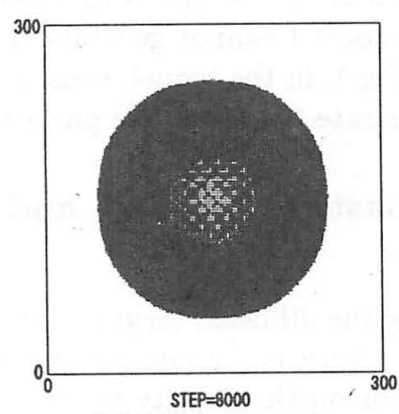

(b) Without noise

Figure 8: Eden-like pattern (Case-5)

\section{Analysis of Influence of An- tibiotic on Bacterial Colony by Simulations}

Generally, the movement and the growth of bacteria are also controlled by antibiotic. Antibiotic plays a role in suppression of growth of bacteria. Therefore, assuming that antibiotic is corresponding to situations of the hard agar and a little nutrient, consider the effect of antibiotic on bacterial colony patterns. Figures 9 and 14 are experimental results of bacterial colony under antibiotic stress and no antibiotic stress (quoted from [5]) with a different initial colony.

Setting as $G=(0,1) \times(0,1), d_{v}=1.0, a_{1}=$ $1 / 2400, a_{2}=1 / 120, \alpha=1, c=0.03$,

【Case without antibiotic:】 $d_{u}=0.07, v_{0}=0.14$

【Case with antibiotic: 】 $d_{u}=0.05, v_{0}=0.087$

and changing initial value $u_{0}$ as below, simulations are performed.

(Case-6) $u_{0}$ is line given by Fig.10.

(Case-7) $u_{0}$ is line and circle given by Fig. 15 .

Results of simulations of Cases 6 and 7 are shown in Figs.11, 12, 16 and 17.

Figure 9 is experimental results of bacterial colony with the initial colony like line.(see Fig.10 in simulations.) Under the initial condition as shown in Fig.10, results of simulations by the model I at Case 6 are shown in Figs. 11 and 12. Both Figs. 11 and 12 are similar to the experimental results in Fig.9. If it were not for noise in Fig.12, the generated pattern would become symmetric (artificial) one as shown in Fig.13 which is not observed in the experiment. So, the stochastic model is very effective as the model of colony formation under anitibiotic and no antibiotic stress.

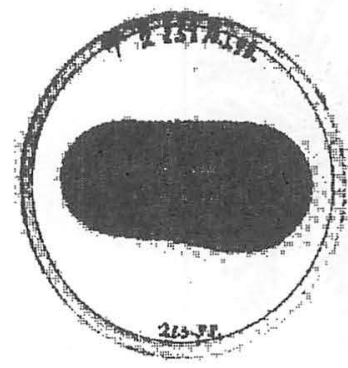

Without antibiotic

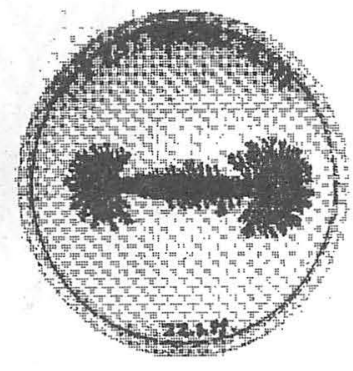

With antibiotic
Figure 9: Experimental results of bacterial colony formation with line colony at $t=0$ (quoted from [5]) 


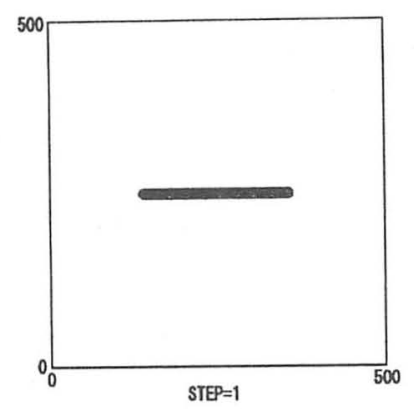

Figure 10: Initial Value in (Case-6)

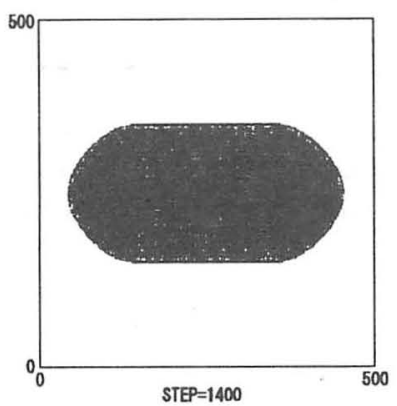

Figure 11: Results of (Case-6) without antibiotic under noise

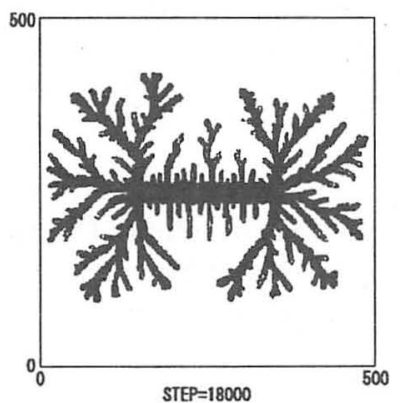

Figure 12: Results of (Case-6) with antibiotic under noise

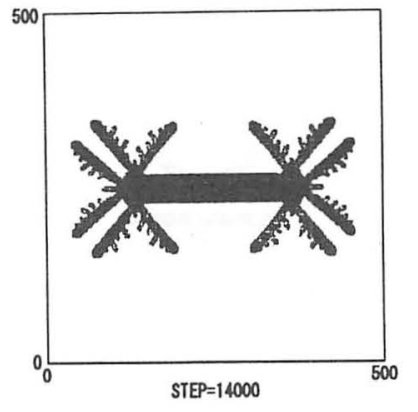

Figure 13: Results of (Case-6) under no noise

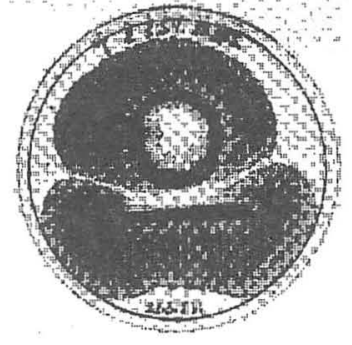

(a) Without antibiotic

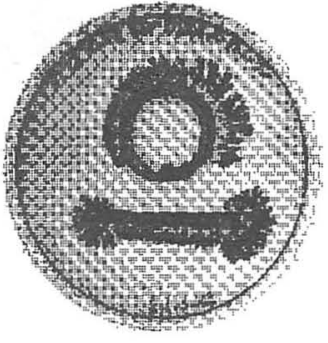

(b) With antibiotic
Figure 14: Experimental Results with Line and Circle Colony at $t=0$ (quoted from [5])

Figure 14 is experimental results of bacterial colony formations with the initial colony like circle and line shapes. (see Fig.15 in simulations) Under the initial condition as shown in Fig.15, results of simulations by the model I at Case 7 are shown in Figs.16 and 17 . Both Figs. 16 and 17 are similar to the experimental results in Fig.14. If it were not for noise in Fig.17, the generated pattern would become symmetric (artificial) one and the colony does not grow from the side of line as shown in Fig.18, which is not observed in the experiment. So, the stochastic model is very effective as the model of colony formations under anitibiotic and no antibiotic stress. So, we can say that the noise plays a very important role in the bacterial colony formation under both antibiotic and no antibiotic. 


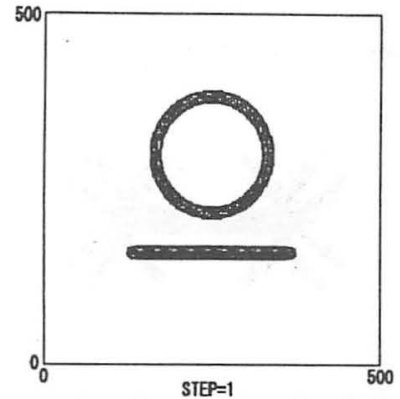

Figure 15: Initial Value

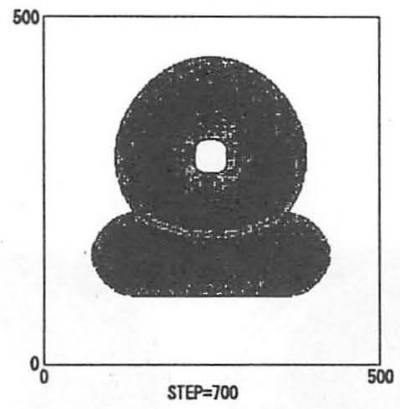

Figure 16: Result of (Case-7) without antibiotic under noise

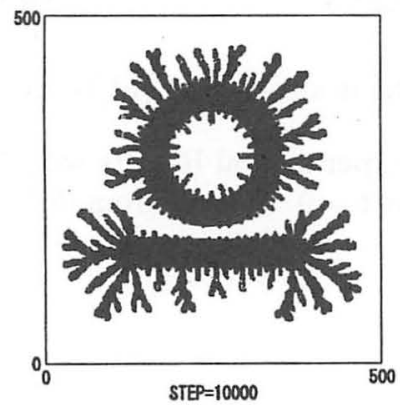

Figure 17: Result of (Case-7) with antibiotic under noise

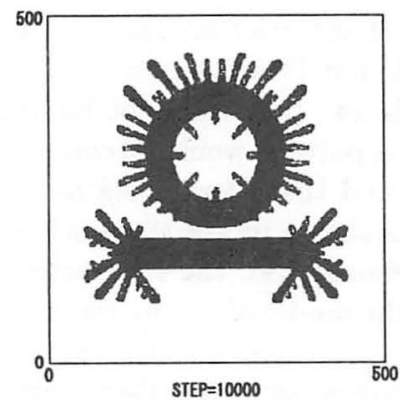

Figure 18: Results of (Case-7) with antibiotic under no noise

\section{Conclusion}

In this paper, the stochastic model, which can generate all kinds of bacterial colony patterns observed in experiments, have been proposed. And by comparing the proposed model with the deterministic model through simulations, we have concluded that the proposed model were better than the deterministic one because the proposed model could generate patterns as observed in experiments, on the other hand, the deteministic model only generate the artificial pattern which does not show real patterns.

The application of the proposed model to the bacterial colony formation under antibiotic stress and no antibiotic one have been considered. In this case, the proposed model could generate the colony patterns as observed in the experiments.

In this paper, although all kinds of bacterial colony patterns observed in experiments are generated by the two models, Model I and II, in order to generate them by only one model, a detailed study of modeling of growth and conversion rate of active and inactive bacteria is needed and this is an important issue in the future in this research.

\section{Acknowledgement}

This research is partially supported by The Ministry of Education, Culture, Sports, Science and Technology under Grant-in-Aid for Young Scientists (B) 14740075 .

\section{References}

[1] M.Mimura, H.Sekiguchi, M.Matsushita : Reaction-diffusion modelling of bacterial colony patterns, Physica A 282 pp283-303, 2000.

[2] J. Wakita, I. Rafols, H. Ito, T. Matsuyama and M. Matsushita: Experimental Investigation on the Formation of Dense-Branching-MorphologyLike Colonies in Bacteria, J. Phys. Soc. Jpn. 10, 1998 pp.3630-3636.

[3] H. Ito, J. Wakita, T. Matsuyama and M. Matsushita: Periodic Pattern Formation of Bacteria Colonies, J. Phys. Soc. Jpn., 68,1999, pp.14361443.

[4] G. Kallianpur and Xiong, J., Stochastic Differential Equations in Infinite Dimensional Spaces, IMS Lecture Notes-Monograph Series 26, Institue of Mathematical Statics, 1995.

[5] E. Ben-Jacob et al., Bacterial Cooperative Organization under Antibiotic Stress, Physica A 282, pp.247-282, 2000. 